\title{
La demostración de la existencia de Dios en la filosofía de Polo
}

The Demonstration of the Existence of God in Polo's Philosophy

Enrique Moros Claramunt

Universidad de Navarra

enmoros@unav.es
RECIBIDO: 24 DE JULIO DE 2016

VERSIÓN DEFINITIVA: 14 DE DICIEMBRE DE 2016

DOI: $10.15581 / 013.19 .41-66$
Resumen: La demostración de la existencia de Dios es uno de los temas cruciales de la filosofía. Su estudio involucra todos los ámbitos y cualquiera de los métodos del pensamiento humano, que enfrentado a lo más alto ha de dar lo mejor de sí. Una breve introducción al proyecto filosófico de Polo nos conducirá a la crítica de las habituales demostraciones de la existencia de Dios y a la exposición de las dos demostraciones propuestas por Polo. Esta exposición se encamina a aclarar el rendimiento intelectual y humano de la filosofía poliana respecto a Dios. Se concluye que ambas demostraciones se muestran válidas y, a pesar de ello, el fin de la existencia personal humana tiene que ver directamente con el amor trascendental.

Palabras clave: Dios, existencia de Dios, demostración, Polo.
Abstract: The demonstration of the existence of God is one of the crucial issues of philosophy. Their study involves all areas and any of the methods of human thought, which faced the top has to give the best of themselves. A brief introduction to the philosophical Polo project will lead to criticism of the usual demonstrations of the existence of God and the exposure of the two arguments proposed by Polo. This exhibition is aimed at clarifying the intellectual and human performent of polian philosophy about God. It is concluded that both shows is valid and, despite this, the end of human personal existence has to do directly with the transcendental love.

Keywords: God, Existence of God, Argument, Polo. 


\section{La DEMOSTRACión DE LA EXISTENCIA DE Dios}

$\mathrm{D}$ ios en cuanto Dios no forma parte de la experiencia inmediata del ser humano ${ }^{1}$. Pero, a la vez, no es posible vivir humanamente sin encontrarse con Dios, y, sobre todo, es imposible pensar sin encontrarse con Dios ${ }^{2}$. La teología natural es, en buena medida, el resultado del esfuerzo filosófico para articular estas evidencias. Sin embargo, los logros alcanzados en la teología natural dependen en buena medida de la calidad filosófica que se pretenda y la que realmente se alcance. $\mathrm{Y}$ eso, a su vez, es una variable ligada a la radicalidad con lo que cada filósofo ponga de su propia humanidad a la hora de pensar ${ }^{3}$.

En el título de este escrito hay tres sustantivos. Cuando se habla de demostración es inevitable que pensemos en la lógica. Cuando Aristóteles desarrolló este saber tenía como objetivo controlar el lenguaje proposicional para enfrentarse al relativismo sofista ${ }^{4}$. Pero inmediatamente la doctrina de la demostración alcanzó una gran madurez en el desarrollo de las matemáticas. La lógica como doctrina para manejar objetos mentales ha resultado una herramienta imprescindible para la exposición de toda ciencia. De ese modo, ciencia y demostración han llegado a convertirse en sinónimos. El saber adquirido debe presentarse de manera tan sólida que todos puedan seguir el itinerario mental que conduce al conocimiento de la verdad. Pero no todo es susceptible de demostración ${ }^{5}$. Toda demostración parte de principios y debe conducir, según un procedimiento reglado, a la conclusión a todos aquellos que puedan comprenderla ${ }^{6}$. Los principios, por tanto, no son demostrables. Asimismo, tampoco las reglas de la demostración son susceptibles de prueba, porque cometeríamos una petición de principio?.

1 "No tenemos experiencia de Dios como tal". El ser, 231.

2 Polo afirma: "advertimos otra dimensión humana que se encuentra constantemente cuando se estudia al hombre a través de la historia: la religiosidad. Desde las protoculturas hasta nuestros días éste es un hecho abrumador... La religiosidad primitiva es muy sencilla, está centrada en nociones religiosas muy puras, en una visión de Dios sumamente adecuada, aunque naturalmente no es filosófica, ni está elaborada de ninguna manera". Quién es el hombre, 225.

3 Cfr. Introducción, 44.

${ }^{4}$ Cfr. Curso de teoría III, 78-79.

${ }^{5}$ Para Polo, "la teoría del silogismo es la lógica del conocimiento de lo necesario. El silogismo es el método demostrativo riguroso. Se requieren dos condiciones para que funcione con entero rigor: que nosotros conozcamos lo necesario y que lo necesario exista”. Introducción, 139.

${ }^{6}$ Esta última apreciación viene a subrayar la precedencia que, según Polo, debe darse a los Tópicos en la lógica aristotélica sobre los Analíticos. Cfr. Introducción, 140.

7 Polo defiende una visión de la filosofía -y del pensamiento- en el que el momento argumentativo no es el principal: "su progreso no consiste estricta y exclusivamente en razonar o en respon- 
La filosofía y, en último término, la metafísica son saberes de principios ${ }^{8}$. La filosofía investiga el principio de la realidad. La metafísica es la ciencia de los primeros principios y de los trascendentales. Así pues, las demostraciones no caben estrictamente ni en la filosofía ni en la metafísica. Pero sin principios tampoco caben demostraciones. La racionalidad no puede limitarse a lo demostrable, salvo que quiera condenarse a sí misma, en último término, a la irracionalidad. Y con ella todo el saber humano sería irracional, y el ser humano acabaría en la cerrazón de su propia soledad’. La demostración exige el saber de los principios y debe conducir eficazmente la luz desde ellos hacia sus conclusiones. Porque sin luz no hay adónde ir y, en consecuencia, tampoco existe ningún camino.

Esta dificultad afecta esencialmente a los otros dos sustantivos mencionados. La existencia o realidad es, sin duda, un principio. El ser, que es el objeto de la metafísica, es el primer principio y sobre él no cabe demostración alguna.

Sin embargo, cuando se habla de demostrar la existencia de algo se ha de partir de la definición nominal de aquello cuya existencia buscamos. Pero si se trata del ser o de la existencia o de la realidad no hay definición nominal posible, puesto que el ser no es ningún contenido mental.

Por otro lado, no podemos aplazar la consideración de que nuestro pensamiento ha descubierto la existencia de muchas cosas que nunca habíamos soñado que pudieran existir. Es decir, nuestro conocimiento del mundo avanza y cada vez sabemos más y mejor de más cosas. Pero en un cierto sentido no se puede progresar en el conocimiento de los principios, porque sin éstos ningún saber podría ser lo que pensamos. Tampoco cabe ninguna duda que hay un avance en el conocimiento de los principios. En esto consiste el progreso de la metafísica. Todo pensador que quiera añadir algo a la historia de esta cien-

der argumentativamente a las críticas. Y no es así, porque el hallazgo es siempre lo primero (la forma más alta de inteligencia es la inventiva; antes de la demostración y de la formulación más rigurosa, está el encontrar). Si el momento demostrativo o reformulativo es el que predomina durante una época, se puede decir que no hay entonces creación filosófica. La filosofía va siempre adelante saliéndose de los datos (ése es, por lo demás, el único test de inteligencia en sentido propio). Si uno se atiene a los datos, no añade nada. Lo más difícil es encontrar una dificultad. Responder a ella es inventar". Introducción, 87-88.

8 "El ser del que se ocupa la metafísica es el ser como principio, o el sentido principial del ser". Presente y futuro, 143.

9 "La forma más moderna de ateísmo: como nosotros estamos afectados por una finitud insuperable, no sabemos qué quiere decir que Dios exista... Nos quedamos en la pura soledad de la existencia". Presente y futuro, 131. 
cia tendrá que tener la pretensión de añadir algo sobre los mismos principios sobre los que llevan pensando los metafísicos desde el día en que Aristóteles alumbró este saber.

Así pues la expresión "demostración de la existencia" está sujeta a una doble dificultad. La existencia es un primer principio sobre el cual no cabe demostración, dado que no es ningún objeto de pensamiento. Por otro lado, es necesario pensar de nuevo con rigor y profundidad los primeros principios. Ese pensar riguroso hace referencia a la ciencia y a la demostración, por mucho que esta última no quepa de ningún modo y eso nos obligue, además, a pensar la ciencia de un modo superior al puramente demostrativo. Esta posibilidad y necesidad de pensar la realidad es la experiencia que justifica la actividad filosófica en su sentido último, es decir, la que permite definir la metafísica como "ciencia buscada". Aunque el concepto de demostración señala un itinerario de progreso del saber, es preciso pensar más hondamente el propio saber. No sólo para saber más sobre el saber, sino también -iy sobre todo!porque necesitamos un conocimiento más pleno de los mismos principios sobre los que se asienta el saber.

Para que la expresión "demostración de la existencia" tenga sentido metafísico, es necesario que se interprete el sustantivo "demostración" de tal modo que no imposibilite el avanzar intelectualmente hacia los primeros principios. De este modo, "demostrar la existencia" llegaría a ser equivalente a "pensar el ser", sabiendo que el ser es justamente aquello que no se puede pensar, o, mejor, que el pensar objetivo sea exactamente lo que hay que abandonar para advertir el ser, la realidad que existe más allá del pensar, la trascendencia. En definitiva, nos encontramos de lleno en una investigación metafísica.

Cuando se habla, además, de Dios todo se complica, a la vez que se hace realmente interesante. "Se trata ahora de enfocar el más importante de todos los temas: Dios. Dios es el Ser respecto del cual nosotros los hombres (aparte de otras muchas cosas) nos jugamos el tipo, por activa y por pasiva... Sin embargo, nuestra capacidad de Dios ha sido puesta en duda"10.

Dios no puede ser un objeto del pensamiento. No puede ser una sustancia más del mundo. Dios no puede ser simplemente el ente más grande. Dios

${ }^{10}$ Presente y futuro, 39. Y, en página siguiente, añade: "Pensar a Dios es pensar por todo lo grande, y esto conlleva una experiencia profunda, espléndida, de nuestro pensar". $\mathrm{Y}$ aún sigue: "Abordar el tema máximo que es, al mismo tiempo, el más arduo, el que mayores esfuerzos entraña: el inagotable conocimiento de Dios". 
no entra a formar parte de ningún conjunto con el resto de la realidad. Dios es el creador, es decir, el primer principio, el origen del universo y de cada persona. Crear es dar el ser, pero eso no puede significar hacer pasar algo que había en algún orden de cosas a la realidad. Si debemos pensar a Dios como creador no se puede suponer nada previo a su misma existencia. Por tanto, la existencia de Dios no puede compararse de ninguna manera a la existencia de alguna otra cosa de la realidad. La existencia de Dios es la existencia de la misma existencia. Por esa razón, la "existencia" en la "existencia de Dios" no puede tener el mismo sentido que la existencia de cualquier ser del universo.

Una prueba de la existencia de Dios no puede pensarse como una expedición a la búsqueda del unicornio. Tampoco Dios es consecuencia de nada, sino el principio de todo. Es el ser originario. En este sentido, la demostración de la existencia de Dios sólo puede consistir en el esfuerzo intelectual para alcanzar el principio de todos los principios.

De acuerdo con el valor existencial de los primeros principios, según Polo, la existencia de Dios es el valor real del principio de identidad. La demostración de la existencia de Dios, entonces, tomará la forma de advertir, alcanzar y presentir el valor real de los primeros principios y explicar su mutua vigencia como primeros principios. Por eso se habla de demostración: porque, de entrada, el hombre no conoce biográficamente los primeros principios como tal y ha de avanzar hacia ellos. Y, segundo, porque ese avanzar requiere de un rigor y coherencia sumos, porque el saber de los primeros principios es el saber exacto, preciso, mostrable, que alcanza todo otro saber y decide sobre el lugar y la importancia de cada uno en la realidad.

\section{BREVE INTRODUCCIÓN AL PROYECTO FILOSÓFICO POLIANO}

En estas páginas se pretende estudiar la demostración de la existencia de Dios en el pensamiento de Leonardo Polo. Pero el pensamiento del filósofo madrileño no comparece sólo como campo de estudio, sino que se pretende hacer filosofía al estilo del profesor Polo. Esto, por su parte, significa que en la obra de Polo convergen el esfuerzo por pensar la realidad y alcanzar la trascendencia con el estímulo necesario para no considerar suficiente repetir lo que ya se ha dicho, sino dar un paso más allá o alcanzar una nueva profundidad. Lo cual no implica, en absoluto, la pretensión de originalidad, sino el esfuerzo de pensar en primera persona tanto como se pueda, sin limitarnos a las formulaciones lingüísticas ya logradas. 
Para comprender el pensamiento de Polo y lograr seguir pensando a partir de él será preciso comenzar por establecer el marco hermenéutico que permita exponer la novedad de su pensamiento y la perennidad de la filosofía que él pretende prolongar ${ }^{11}$. El propio Polo comienza El acceso al ser con una sentencia de santo Tomás en el comentario a los libros aristotélicos de la Metafísica, según la cual es conveniente antes de comenzar a filosofar, es decir, a la hora de desarrollar una consideración universal de la verdad, desarrollar una duda universal acerca de ella, y, de este modo, tomar en cuenta todas las dificultades $^{12}$. El hombre no comenzó su existencia haciendo filosofía, sino que la aparición de la misma es un fruto maduro del esfuerzo humano por vivir humanamente. Vivir como hombres significa principalmente crecer. Y cada persona debe aportar lo que pueda para ayudar a crecer, antes que nada, creciendo personalmente. La filosofía no puede entenderse como un conjunto de tesis que sea necesario sostener, sino que el proyecto de la vida teórica consiste en crecer en el conocimiento de la realidad, porque ése es el modo de vivir en la verdad para los hombres. "Se es filósofo como servidor de la verdad, como amante y realizador de ella" ${ }^{13}$. Sólo alcanzando la verdad que inspira el hombre, éste puede hacer el bien que da sentido a su existencia.

La pretensión de alcanzar lo radical de la realidad es también la primera formulación de la dignidad del hombre ${ }^{14}$. Pero el ser humano puede hacer algo más que pensar la verdad: puede amarla y dejarse inspirar por su belleza ${ }^{15}$. Polo considera que en el recorrido histórico de la filosofía hay dos hitos imprescindibles: Aristóteles y Tomás de Aquino. Aristóteles descubrió el concepto de acto, que ha sido decisivo tanto para pensar la realidad como para explicar el conocimiento humano. Tomás de Aquino, por su parte, pensó la distinción real de esencia y ser, que lleva adelante el descubrimiento aristotélico y permite una formulación precisa de la diferencia entre el Creador y la criatura y de lo característico de la persona ${ }^{16}$. Pero la filosofía no puede detenerse en averiguaciones ajenas o en repetir temas y fórmulas ya establecidos. Como activi-

11 "Ninguna doctrina metafísica surge del vacío, sino que nace situada en la historia. Gracias a eso puede aspirar a traer algún avance o alguna novedad”. El acceso, 290.

${ }_{12}$ Cfr. El acceso, 15.

13 Introducción, 45.

${ }^{14}$ Cfr. Introducción, 38.

15 Cfr. Introducción, 46.

${ }^{16}$ Polo afirma que en su pensamiento "lo más importante es mantener la primacía del acto de ser". "El descubrimiento de Dios desde el hombre", en Studia Poliana, 1 (1999), 14. 
dad humana exige siempre lo mejor de nosotros y pide nuestra energía intelectual completa para seguir pensando. Se trata de pensar la realidad según nuestra propia altura histórica ${ }^{17}$.

Nuestro tiempo está decisivamente marcado por el desarrollo de la filosofía moderna desde Descartes a Hegel y a Heidegger. Lo decisivo de esta etapa es el descubrimiento de la singularidad humana: el hombre no es simplemente algo real, sino sujeto. Sin embargo, en su virtud se encuentra también su limitación: quisieron pensar el sujeto como principio, de modo simétrico a como los griegos consideraron la sustancia. Pero eso es exactamente lo que una persona no puede ser. Por esa razón aunque no hayan alcanzado una comprensión adecuada de la realidad, no podemos dejar de lado sin más su inspiración. Hacer filosofía de acuerdo a nuestra altura histórica requiere tanto recuperar el pensamiento metafísico como pensar de modo congruente la dignidad y el ser de la persona. La metafísica debe continuarse con la antropología trascendental: no basta estudiar el ser principial, sino que debemos alcanzar un conocimiento propio del ser humano desde el que se iluminarán, a su vez, algunos temas propios de la metafísica ${ }^{18}$. Por eso, "hay que continuar la filosofía"19 ya que "la filosofía siempre es capaz de ir adelante, siempre se puede saber más" ${ }^{20}$.

El modo concreto que Polo descubre para realizar ambas tareas es lo que él mismo llama "el abandono del límite mental". En la medida en que la filosofía es metafísica, debe pensar la realidad, el ser. Para eso es preciso advertir que nuestro pensamiento operativo no sirve, porque siempre que se piensa $y a$ se ha pensado. Pensar significa poseer en presencia lo pensado. El modo intelectual de poseer la realidad es eximirla de su propio ser para que adquiera su carácter de objeto. "He afirmado con insistencia que no cabe idea del ser" ${ }^{\prime 2}$. Pero si algo ya se ha pensado, no cabe proseguirlo. Además de ese modo de pensar, debe haber otro por el que podamos advertir que ser pensado y ser real son radicalmente diferentes. Y captar esa diferencia es exacta-

17 Porque "la filosofía tradicional... ha quedado detenida en lo que respecta a la altura histórica. Y eso sí que es una falta, precisamente la falta de perennidad... porque hay que superar el enmarañamiento consciente... la necesidad de proseguir sigue acuciando. Por eso, hacer hoy metafísica es advertir esta necesidad de nuevo, otra vez, en el carácter histórico -acrecentamiento- de su perennidad". El acceso, 291.

18 Cfr. El acceso, 293.

${ }^{19}$ Introducción, 41.

${ }^{20}$ Introducción, 68.

${ }^{21}$ El ser, 166. 
mente la condición para hacer metafísica. Esa diferencia es exactamente el descubrimiento aristotélico del acto o actividad. Dicho con una sencilla fórmula que el profesor Polo atribuye a Moore: "conocer una elipse no es una elipse"22. Además del pensamiento objetivo, que resulta decisivo para la vida práctica del hombre y en el uso del lenguaje, debe haber otro modo de pensar, que, siguiendo también una expresión clásica, Polo denomina "hábito o virtud intelectual".

Desde ese punto Polo acierta a proponer la realidad humana como intelectual. Para eso toma pie de otro concepto aristotélico decisivo: el intelecto agente. Pensar es una forma de ser diferente, es una realidad distinta de la que hablaba la metafísica clásica. Un ser capaz de pensar es una realidad diferente a la actividad del universo. Así seguimos la inspiración moderna. Pero, a diferencia de ella, Polo suele repetir: "el yo pensado no piensa", porque lo pensado es eximido de pensar por el mismo pensamiento. El hombre no puede ser el fundamento de su pensamiento, no es el sujeto moderno. Sino que la realidad humana es aquella que existe además de la realidad del universo. Por eso la antropología, en la medida que estudia la realidad del hombre, no es la ciencia del ser, sino la del co-ser, la realidad que consiste en co-ser con el mundo, los demás y la trascendencia. Y su tarea primera será descubrir y formular de modo coherente los trascendentales personales. "La persona es co-existir -íntimamente abierta-, libertad, luz intelectual, amor donal. Éste es el elenco de los trascendentales personales" ${ }^{23}$.

Esta diferencia entre realidades se advierte precisamente en la existencia de virtudes intelectuales. El intelecto agente no cumple sólo la función de universalizar las especies sensibles para que el intelecto paciente pueda pensar los conceptos, sino que su actividad permite que las facultades crezcan en su misma realidad activa y, de este modo, sean capaces de más. Ese más son las virtudes o hábitos intelectuales. De este modo, Polo destaca el error antropológico de juzgar al ser humano por lo que sea capaz de lograr: el principio del resultado ha de sustituirse por la realidad del crecer íntimo ${ }^{24}$.

Este resumen sucinto del proyecto de Polo indica las líneas generales en las que se inscribe su teología natural y, antes que nada, la demostración de la existencia de Dios. "El tema de Dios, atracción y acicate decisivo del saber, se

22 Nominalismo, 211.

23 Epistemología, 194.

${ }^{24}$ Cfr. Presente y futuro, 94-96. 
abordará en todos ellos" ${ }^{25}$, es decir, en todo saber que se puede lograr a través de todas las dimensiones posibles del abandono del límite mental. Tanto la metafísica como la antropología deben considerar la existencia de Dios. La teología natural es el culmen del conocimiento filosófico.

De este modo se obtiene a la vez la valoración positiva de las demostraciones de la existencia de Dios y las limitaciones que cada una presenta. La cuestión de la existencia de Dios es el tema que requiere mayor esfuerzo filosófico y, en consecuencia, permite una perspectiva decisiva para valorar cada filosofía. De la misma manera, la mayor verdad que busca la filosofía no puede concluir en un concepto. No hay una última verdad que podamos poseer o, de otro modo, la filosofía no concluye en ninguna proposición que exprese todo lo que se puede conocer. La filosofía es, por tanto, inacabable y requiere una audacia infinita: pensar más y mejor lo más alto, sabiendo que supera todo lo que podemos pensar. El futuro del hombre depende del atrevimiento para pensar a Dios ${ }^{26}$.

\section{Las dos demostraciones de la existencia de Dios}

Según Polo, caben demostraciones de la existencia de Dios tanto en metafísica como en antropología, porque ambas son ciencias trascendentales. Pero, en cuanto ambas tratan de lo primero y superior, no pueden partir de postulados y avanzar hacia las conclusiones, sino que deben alcanzar una comprensión coherente de los principios. Ambas han sido desarrolladas por el filósofo madrileño en El ser Iy en El descubrimiento de Dios desde el hombre. Y am-

${ }^{25}$ El acceso, 295. Se trata de la última frase del libro programático de Polo. En el tomo II del Curso de teoría del conocimiento Polo dice: "La noción de Dios aparece en todos los niveles operativos de la inteligencia, y en sus hábitos. Si la inteligencia no fuese operativamente infinita, no se podría 'nocionalizar' a Dios. Por tanto, Dios es el máximo pero no la noción máxima, lo cual indica que la noción de Dios se ha de proseguir con la profundización en Dios. Por eso hay que demostrar la existencia de Dios", 169.

26 "Pensar a Dios es pensar por todo lo grande, y esto conlleva una experiencia profunda, espléndida, de nuestro pensar. Si el pensar queda reducido a un apéndice sucedáneo, puramente pragmático, a una especie de instrumento desvitalizado, se compromete el acceso a Dios. No perdamos de vista lo que decíamos antes: el tema de Dios, desde el punto de vista del conocimiento, es solidario del carácter vital del conocimiento. Dios es Dios de vivos; si nuestro pensamiento piensa a Dios, es porque está vivo para Dios: si no, no lo piensa. Un pensamiento disecado, como un insecto etiquetado y clavado con un alfiler, encerrado en una cajita, no piensa a Dios, o lo hace de una manera tan precaria que se confunde con pensar cualquier cosa. Tomás de Aquino, que emplea a veces la ironía, insiste en el tema al hablar del conocimiento 'imperfecto de lo imperfecto': la acumulación de dos imperfecciones. Por el contrario, en la medida en que nuestro pensamiento se viviente -y solamente en esa medida-, nuestro itinerario mental in Deum será un camino gallardamente recorrido". Presente y futuro, 40. 
bas han sido analizadas y desarrolladas en sendos artículos ya publicados ${ }^{27}$. Ahora interesa más bien poner de relieve el esfuerzo filosófico de Polo para pensar a Dios desde y a partir de las demostraciones, primero desde la metafísica y, después, desde la antropología trascendental.

\section{a) La demostración metafísica de la existencia de Dios}

En primer lugar, Polo considera que "del abandono del límite se desprende también una importante ganancia para el tema de la demostración de la existencia de Dios, Ser Increado, que alcanza congruencia metafísica" ${ }^{28}$. En estas líneas se establece lo que el nuevo método metafísico propuesto permite en primer lugar: la congruencia. A la vez, se afirma explícitamente que dicha congruencia permite una ganancia para el tema de la demostración de la existencia de Dios. A mi entender la palabra clave es "increado". Es bien sabido que las demostraciones de la existencia de Dios son de algún modo simétricas con las demostraciones de la creación. Increado equivale a Creador. El elemento negativo que aparece en "increado" subraya la diferencia entre Creador y creación. Si el abandono del límite supone un desarrollo posible de la metafísica, eso significa que esta ciencia nos permitirá alcanzar una visión nueva de lo creado. Y, a su vez, esa comprensión de lo creado permitirá entrever una mayor congruencia con lo Increado, con el Creador.

El problema que tiene en mente Polo es la dificultad de pensar la diferencia entre Dios y los seres, que presentan tanto el pensamiento clásico como el contemporáneo. Este problema se puede condensar en una aporía: o bien Dios se distingue de lo creado porque en lo creado se distinguen realmente ser y esencia, mientras que en Dios su esencia se identifica con su ser y, de esta manera el ser de Dios se identifica con el mismo ser de las criaturas; o bien el ser de las criaturas consiste en una degradación del ser divino ${ }^{29}$. La primera

${ }^{27}$ Cfr. S. PIÁ TARAZONA, "De la criatura a Dios. La demostración de la existencia de Dios en la primera dimensión del abandono del límite mental”, en Anuario Filosófico, 1996 (29), 929-948; y E. R. MOROS, "La demostración de la existencia de Dios a partir de la libertad", en Anuario Filosófico, 1996 (29), 805-814.

${ }^{28}$ El ser, 21.

${ }^{29}$ En otra ocasión Polo describe la consecuencia de su pensamiento a este respecto: "Se quita de en medio una noción que es común (de acuerdo con la simetría ya señalada) a clásicos y modernos, que es la noción de causa primera. Los modernos descubren que la noción de causa primera equivale a la de espontaneidad. Para los clásicos... conlleva degradación de la causalidad en lo creado". Nominalismo, 231. 
parte no hace justicia a la trascendencia del absoluto, la segunda no hace justicia a la creación divina. La aporía se supera si conseguimos pensar en Dios como ser y creador del ser y, por tanto, si distinguimos entre diversos actos de ser. Por esa razón, el abandono del límite que permite pensar el ser tiene un rendimiento excelente para pensar el ser de $\operatorname{Dios}^{30}$.

La congruencia de la que habla Polo es la congruencia de los primeros principios o del ser principial. Los primeros principios son tres: identidad, nocontradicción y causalidad. Son principios existenciales, es decir expresan la actividad propia del ser que puede describirse como tal ${ }^{31}$. Precisamente por eso son principios metafísicos. La no-contradicción expresa el ser que comienza y no cesa ni es seguido. La identidad expresa la actividad propia del ser originario $^{32}$. La causalidad expresa el ligamen de la no-contradicción con la identidad. La búsqueda de la congruencia entre ellos es exactamente el esfuerzo filosófico por pensar a Dios. Así lo explica el propio Polo: "En orden al ser principial, el intelecto es el conocimiento del ligamen transcendental. A esto se ha llamado durante milenios, con toda exactitud, demostración de la existencia de Dios. En orden al núcleo, el intelecto es el logos como búsqueda: es el famoso desiderium naturale videndi Dei, deseo ineficaz porque la búsqueda depende de la futuridad libre..., nadie puede demostrar la existencia de Dios si no la busca. Esta búsqueda, aunque incapaz de intuición, es particularmente no arbitraria... Y con todo, la búsqueda como tal es incondicional: no es la búsqueda de algo, sino buscar-se. Si se prescinde de esta búsqueda, el logos se angosta decisivamente" ${ }^{{ }_{3}}$.

Aquí se reúne el carácter intelectual del hombre al que corresponde la naturalidad de la filosofía, su carácter de ciencia buscada, la imposibilidad de

\footnotetext{
30 "El pensamiento cristiano considera que es más radical la distinción entre ser y nada... Si el ser se distingue de la nada en sentido real, todavía hay una distinción mayor: aquella que existe entre lo que se distingue de la nada y Dios". Y, a continuación, afirma que Dios es "la radical trascendencia" y "de suyo, la criatura es nada". Por lo tanto, también: "Aquel de quien depende la diferencia radical entre el ser y la nada es omnipotente, y asimismo, misericordioso, pues la creación no es un descenso ontológico necesario". Y, finalmente, "para ella [la criatura] ser es mantenerse en el ser". Presente y futuro, 133-134.

31 "Si los primeros principios son reales y el hábito correspondiente tiene que ver con el intelecto agente, ese hábito será el conocimiento del ser... El hábito de los primeros principios es el conocimiento en acto de los primeros principios, y los primeros principios son actos, son reales, por eso el conocimiento de los primeros principios es el conocimiento del actus essendi: el desarrollo, el despliegue de la teoría del actus essendi de Tomás de Aquino”. Nominalismo, 171.

32 Esta afirmación es preciso complementarla, como se hará más tarde, con un motivo decisivo: "Dios es el Origen y, por tanto, simplemente Incausado". Nominalismo, 223.

${ }^{33}$ El acceso, 111-112.
} 
su completitud y el modo peculiar en que la metafísica es una ciencia estricta sin que sea posible la intuición de Dios. Dicha imposibilidad de intuir a Dios significa su superioridad sobre toda la actividad intelectual del ser humano: Dios, como el mismo ser, no puede ser objeto de pensamiento. De este modo, la actividad inteligente sobre Dios apunta a la libertad del amor aceptante del don que me ofrece lo que es más íntimo a mí que yo mismo y así puede mostrarme quién soy yo, es decir, en quién encuentro mi réplica, que es imposible lograr en el propio pensamiento. $Y$ aquí se encuentra otro engarce, continuidad y progreso entre metafísica y antropología.

Vale la pena detenernos en una descripción del problema de la existencia en el racionalismo, en relación con el argumento ontológico. Polo sostiene que si Dios es una esencia necesaria, esto es, que tiene en sí mismo la razón de su propia existencia, entonces no es posible pensar el ser divino. De este modo, se sigue "concibiendo la existencia como la realidad extraesencial de la esencia. Lo decisivo es que la esencia divina necesita existir, que no se ha barrido totalmente de su realidad la existencia empírica y que, por lo tanto, la realidad, como pura realidad esencial exhaustiva, no ba sido considerada" ${ }^{34}$.

Este texto concluye con la crítica decisiva de Polo a la demostración ontológica de la existencia de Dios. Pero, a la vez, señala la clave existencial que Polo considera necesaria para pensar originariamente en metafísica y, por tanto, también en la teología natural. Si la existencia que se busca es la existencia de algo, entonces no podremos alcanzar de ninguna manera el carácter existencialmente activo del ser. Porque o bien el ser es el mero hecho de darse, pura existencia empírica, o bien, es una posición impensable, puesto que lo que puede pensarse es, por definición, la esencia. Es decir, considerar el ser como "la realidad extraesencial de la esencia" es la expresión patente de que no se sabe nada del ser real, más allá del pensamiento. Y no se puede añadir nada a la metafísica.

Por esa razón, aquí se encuentra el núcleo de la aportación poliana: "Si esta distinción de campos (entre el ser real y la idea) no se lleva a cabo es imposible impulsar la investigación hasta la demostración de la existencia divina. Demostrar la existencia divina equivale a advertir, precisamente, la inteligibilidad de la persistencia fuera de la mente bumana" ${ }^{35}$. La inteligibilidad de la persistencia señala exactamente la actividad real del ser finito. No se trata sólo de

${ }_{35}^{34}$ El acceso, 162.

35 El ser, 166-167. 
que podamos conocer la realidad, sino que podemos advertir intelectualmente el ser mismo de la realidad. "Si la existencia no es una idea, habrá que averiguar qué significa no ser idea comenzando fuera del orden de las ideas" ${ }^{36}$. Que el ser real no sea idea, no quiere decir que no sea inteligible. Es inteligible en la misma medida en que es inteligible el principio de no-contradicción.

Por esa razón, la adecuada comprensión del principio de no contradicción permite una lectura más precisa de la identidad. "Al decir que el ser no es idea se configura inmediatamente esa otra fórmula: 'el ser es el ser'. Lo que se elimina con el comienzo trascendental es el sentido predicamental de esta fórmula" ${ }^{37}$. Y de ese modo se advierte a la vez que el principio de identidad es también inteligible. Es la inteligibilidad del ser originario. Se trata del elemento cognoscitivo de carácter afirmativo necesario para que nuestro conocimiento de Dios no sea meramente negativo.

Aquí se puede subrayar cómo el progreso de la metafísica conduce a una profundización en la teología natural. La razón es sencillamente expuesta por Polo: "la creación es un efecto de Dios y, por lo tanto, se puede probar la existencia de Dios desde ella" ${ }^{\text {8 }}$. Cuando el pensamiento consigue zafarse de las ideas, en el momento en que se comprende que el ser real es actividad, entonces necesariamente el ser divino no puede ser un mero conglomerado de atributos perfectivos, sino la actividad misma originaria. Que yo sepa hasta ahora nadie se había atrevido a tanto. Ciertamente no hay idea de Dios: ya no es necesario demostrar que lo alcanzado es Dios, porque sólo Dios es el ser originario, su acto es el valor existencial del principio de identidad y no demostración de la existencia de algo.

Por esa razón, aunque las demostraciones de la existencia de Dios no pueden sencillamente poner delante de nuestros ojos la existencia divina, no pueden equiparar la existencia divina a la existencia del universo, no pueden ser la existencia de "algo", no se puede decir que Dios no sea inteligible. Además, Dios no es una esencia que ocurre que existe. Dios es actividad existencial originaria. Demostrar la existencia de Dios significa encontrar un modo de apuntar a lo inalcanzable en cuanto tal. Polo lo dice con su originalidad característica: "Dios es el inasible Ser originario. Tanto como lo que es, importa en Dios, por así decirlo, el modo como lo es, porque este modo es

\footnotetext{
${ }^{36}$ El ser, 167.

${ }^{37}$ El ser, 167.

${ }^{38}$ Curso de teoría II, 87.
} 
constitutivo de 'lo que es': en Dios la identidad, en su realidad originaria, tiene el sentido de rebasar el haber, y con él, todas las coordenadas en que quiera inscribirlo la mente humana... No es que Dios sea sin comprender, sino que el ser divino no tiene como requisito algo... Con otras palabras: al comprenderse, Dios es en el modo de ser, no en el modo de ser conocido. La esencia divina es la identidad. La identidad significa el carácter de origen" ${ }^{39}$.

Polo propone un modo de pensar filosófico que permita conocer más profundamente a Dios. El abandono del límite mental es el modo de pensar lo que supera el orden predicamental y la consistencia propia de la sustancia aristotélica. Se trata de pensar el acto, pero no como idea general o como concepto abstracto, sino como actividad real. Pensar con rigor, es decir, de modo congruo y suficientemente descriptivo el ser y los principios primeros del ser. "Dios supera toda consistencia. Por decirlo así, la altura en que está colocado el tema de la existencia de Dios rebasa tanto la predicación del ser como el consistir en ser" ${ }^{\prime 0}$. De ese modo se puede pensar a Dios más allá de las limitaciones de la filosofía de la naturaleza y se sitúa el tema exactamente en el punto cenital del pensamiento humano. "El carácter de origen señala que, con plena prioridad, Dios es todo sin predicación ninguna. El carácter originario 'define' la esencia divina. En estrecha correspondencia, en Dios no hay faceta analítica alguna, ningún predicado es realmente discernible en el origen... Ninguna teoría humana está en condiciones de decir lo que significan los atributos divinos como ser" ${ }^{\prime 1}$.

Dios no puede ser ningún resultado, no puede formar ningún todo con lo creado, sino, por el contrario, es origen, identidad originaria. "En principio, y dicho de forma indicativa, la identidad real, la plenitud originaria, es totalmente: ser todo el ser, sin suspensión ni distinciones" ${ }^{\prime 2}$. No es posible, por tanto, una analítica del origen. La creación es un acto de la bondad generosa de Dios, no un predicado que quepa atribuir al origen. "Dios es Origen. Esta expresión no es predicativa o esencial, sino existencial, y a ella se llega, sin duda alguna, por medio de una investigación de índole metafísica” ${ }^{\star 3}$.

39 El acceso, 257-258.

40 El acceso, 249.

${ }^{41}$ El acceso, 249. Polo precisa el orden de la realidad: "El Origen no consiste en identidad. La identidad pertenece al Origen. Tal pertenencia no significa clausura o aprisionamiento, sino desbordamiento originario. La identidad que pertenece señala la superabundancia como intensidad y simplicidad", El ser, 249.

${ }^{42}$ El ser, 225.

43 El ser, 226. 
Podemos indudablemente predicar de Dios el pensamiento, la voluntad, la providencia, etc. Pero esa predicación no expone el sentido del ser divino y está más allá de nuestra capacidad cognoscitiva averiguar el sentido divino de dichos predicados. Por eso afirma Polo: "Efectivamente, se puede probar que Dios existe y demostrar su existencia como Origen, pero en ninguno de los dos casos se puede decir algo de la Existencia de Dios. Cabe afirmar también, en la línea de las consideraciones esenciales, que se trata de Alguien bueno, verdadero, sin medida, único, etc.; mejor dicho, que Dios tiene que ser todo eso. Pero falta en absoluto la posibilidad de contemplar, como contenido mental, lo que tales atributos son en Dios... Para creer en el 'estilo' divino de estos atributos, hay que acudir a la revelación viviente, cuya cima es el Señor. La mente no da nada en que creer y no podemos confundir a Dios con la idea que de Él tenemos. Lo cual no significa sólo que no se puede creer en lo que se comprende, sino, más en el fondo, que el objeto de razón no constituye una noticia íntima de la Existencia de Dios, ni nos da el sentido preciso de lo que ignoramos... Demostrar la existencia de Dios no es lo mismo que encontrarla como resultado dado en la demostración misma" ${ }^{4}$.

De este modo, el conocimiento de la existencia de Dios nos muestra, a la vez, la grandeza de nuestra ignorancia, el elemento negativo imprescindible para pensar a Dios sin reducciones ni antropomorfismos. Considero que este tema es tan importante que la frase con la que Polo concluye la investigación de los primeros principios en Nominalismo, idealismo y realismo dice sencillamente: "la identidad es aquello en que nunca podemos acabar de penetrar" Esto ilumina también la interna estructura de las vías clásicas para demostrar la existencia de Dios. Polo sostiene que "las pruebas que suponen la idea de Dios se estructuran en dos fases. La primera es la prueba misma como proceso - o conexión de procesos- de la mente, cuyo vigor y validez se pueden examinar y juzgar desde un punto de vista lógico, y mediante los cuales se alcanza con seguridad algún existente. La segunda es la mostración de que lo alcanzado por la argumentación puede llamarse Dios. Esta segunda fase es inexcusable, hasta el punto de que todo el problema del valor de la prueba estriba en la conexión de sus dos fases. La corrección formal de la argumentación no prejuzga de suyo nada al respecto. Pero, por otra parte, la segunda fase no es suficiente por sí sola. Por eso, si la existencia divina misma se quiere al-

\footnotetext{
44 El ser, 230.

45 Nominalismo, 233.
} 
canzar como un contenido inferido desde otros, la prueba es incompleta por falta de estructura formal: en ningún caso la idea de Dios asume el rigor formal de la argumentación ni se deriva de ella" "

Por esa razón, -sigue diciendo Polo- "las pruebas supositivas no aspiran a entender, ni siquiera mínimamente, el sentido de la existencia divina. En dichas pruebas, 'Dios existe' significa 'existe de hecho'. El existente se entiende solamente según determinaciones esenciales" ${ }^{\text {" }}$. En ese sentido las demostraciones clásicas de la existencia de Dios suponen una religión desarrollada y el deseo de purificarla de sus elementos antropológicos. En cambio, para Polo, la demostración de la existencia divina pretende alcanzar, o "comenzar a entender a Dios de acuerdo con el sentido de la demostración misma"

Entender el mundo como comienzo trascendental, que ni es seguido ni se acaba, permite vislumbrar el origen. Por eso el comienzo de la demostración es la criatura, como reconoce paladinamente Polo: "La criatura es el respecto demostrativo de su existencia (de Dios)" ${ }^{49}$. Ése es el punto de partida que determina de antemano el objetivo a alcanzar con la demostración. "La identidad es realmente originaria: puesto que se advierte la existencia, y puesto que la existencia como persistencia equivale al comienzo, el abandono del límite mental debe culminar en la identidad real, es decir, en un sentido del es que no sea ideal y al que no se llegue separando, porque en otro caso la distinción entre el ser y la idea se perdería o debería reiterarse, lo cual es incompatible con la persistencia. Éste es el esquema de la demostración de la existencia de Dios. Se notará que a esta demostración le es propia la imposibilidad de pensar previamente lo demostrado. La existencia a la que corresponde originariamente el es está completamente fuera del orden ideal humano. No se trata de demostrar algo. La existencia de Dios sólo es accesible (en la primera demostración del abandono del límite) atendiendo a lo que llamo persistencia. Asimismo, la causalidad en sentido trascendental (y no predicamental, físico o reflexivo) tampoco es separable de la persistencia" ${ }^{50}$.

${ }^{46}$ El ser, 160.

${ }^{47}$ El ser, 160.

${ }^{48}$ El ser, 160.

${ }^{49}$ El ser, 216.

${ }^{50}$ El ser, 168. "Advertida la criatura, Dios no es una conclusión lógica sino un Ser vivo. A partir de la criatura no se dibuja un orden de necesidades lógicas, con alguna de las cuales Dios se alcance. No es éste el sentido de la demostración de la existencia de Dios. Más aún: en la demostración la criatura no funciona como un supuesto, base de conclusiones, sino como un necesitado de explicación, explicación que es todo el Ser divino. La criatura no fundamenta necesidad divi- 
El principio de no-contradicción es un primer principio. El principio de identidad es también un primer principio. Pero pensar los primeros principios exige considerar también la causalidad como primer principio. Así, parece que regresamos a las demostraciones clásicas, siempre que entendamos demostración en sentido amplio como congruencia. Polo advierte la situación y señala la novedad del planteamiento: "La demostración de la existencia de Dios es una denotación causal de la advertencia de la existencia" ${ }^{\text {". }}$. De esta manera, la causalidad no es tampoco una idea, sino la referencia trascendental de la nocontradicción a la identidad. Es la advertencia del carácter existencial de la dependencia del origen: no se trata de demostrar la existencia de una cadena causal que responda a una idea de causa finita propia de la física, sino advertir que la causalidad es también un primer principio que se ha de conocer de modo coherente con los demás primeros principios. De esa manera no se puede plantear el paso de la imposibilidad de una cadena infinita de causas causadas.

Aquí cabe considerar la diferencia clave con las demostraciones clásicas de la existencia de Dios. "Que la existencia de Dios se puede demostrar en virtud de la advertencia de la persistencia y no en virtud del valor de los procesos mentales o de algún principio lógico. La demostración de la existencia de Dios no debe confundirse con la prueba de la proposición 'Dios existe'. Esta última es posible sin abandonar el límite mental" ${ }_{2}$. Pero el coste es alto: "Ahora bien, lo que distingue una demostración instalada en el ámbito extramental de la que se intenta en el plano ideal, no es una diferencia de vía que conduzca a idéntica meta, sino una diferencia en aquello que se entiende ser el tema de la de-

na alguna; no podríamos decir que alcanzamos a Dios en virtud de una razón suficiente de postulaciones que se dan en la criatura -esto es contradictorio con la noción misma de criatura-; sino que, en orden a la criatura, el Ser divino es entero, precisamente por la imposibilidad de admitir una determinación parcial de Dios en función de la criatura. Nótese que en cualquier otro sentido no podría hablarse de demostración de la existencia de Dios... la necesidad del Ser divino debe verse en orden a la realidad creada; sin esa necesidad la realidad creada no es, no ya posible, sino real. Entre Dios y la criatura no hay conexiones lógicas, sino que Dios es el Ser que, siendo, hace-ser, da el ser. Por eso la criatura demuestra rigurosamente la existencia de Dios". La persona, 160.

51 El ser, 168.

52 El ser, 175, nota 33. En cambio, Polo rechaza el argumento ontológico, en la medida en que parte de la mera objetividad. "La existencia de Dios no se puede demostrar a partir de la objetividad; de otro modo sólo se llega a la existencia empírica, y no hay tal existencia empírica de Dios. En último término, éste es el defecto del segundo elemento del argumento de san Anselmo. Se piensa que si se logra determinar la esencia de Dios, en ella se encontrará la necesidad de su propio existir. Con lo cual, aunque lo que se intenta es establecer las implicaciones de la esencia infinita, lo que resulta es una débil comprensión de la existencia”. El ser, 215. 
mostración. De hecho, la noción de Ser Incausado no es una determinación esencial cuya existencia venga asegurada por la realidad de la criatura" ${ }{ }_{3}$.

Así lo que propone Polo es otro camino metafísico, no elaborar una vía más, digamos la sexta, sino una auténtica demostración metafísica, cuyo objetivo apunta directamente al carácter existencial propio de Dios. "La demostración de la existencia de Dios es la culminación del carácter extramental del abandono del haber" ${ }^{\prime 4}$.

Por eso se trata de una demostración sin aparentes pasos lógicos, exenta por tanto de la necesidad lógica propia de un argumento: la necesidad de Dios se muestra no por la fuerza de motivos lógicos, sino por la profundización del pensamiento en la realidad, en el ser y así, como dice Polo, "responde a la consideración primaria de la existencia extramental" ${ }^{5}$. Por eso es un asunto estrictamente metafísico. La inquisición metafísica se orienta definitiva y decisivamente hacia Dios. Sin el origen, el comienzo quedaría en el aire y no podríamos entender por qué lo creado es causa. La advertencia intelectual de la actividad existencial perdería consistencia y acabaría careciendo de valor porque no podríamos entender congruentemente los primeros principios. A la vez, la consideración de los principios de no contradicción e identidad requiere la consideración del principio de causalidad. "La causalidad se define, en la advertencia, como el modo de la referencia del principio de no contradicción a la identidad. Tal modo no es la relación de una esencia a su existir propio. Por lo tanto, la existencia de Dios es demostrable" ${ }_{56}$.

De este modo, se pone también de manifiesto otro aspecto de la particularidad de la demostración poliana de la existencia de Dios: "La penetración

${ }^{53}$ El ser, 216. En este punto se cifra la principal crítica al pensamiento metafísico moderno y contemporáneo sobre Dios: "Demostrar la existencia de Dios no es lo mismo que encontrarla como resultado dado en la demostración misma. Confundir ambas cosas no es más que una consecuencia del prejuicio que ha esterilizado para la metafísica a la filosofía idealista, a saber, la absolutización del haber, el postulado de que la realidad depende de la consistencia”. El ser, 230-231.

${ }^{54}$ El ser, 222.

${ }^{55}$ El ser, 216.

56 "A su vez, la causa causada es la demostración de la identidad originaria. En rigor, la existencia de Dios es demostrada por el acto de ser creado. Según el hábito de los primeros principios, se advierte la no culminación de la persistencia, y causa causada equivale a esa demostración (por eso, el planteamiento no es ontologista). Se va de la existencia creada al Creador; pero, para eso, es menester que la existencia creada no sea efecto. Y para que la causa sea, es menester que sea causa causada, y que causa como primer principio, no signifique otra cosa que causa causada. Insisto: causa causada significa la referencia al Origen que la persistencia comporta. Si la persistencia no comportara esa referencia, sería ultimada; y si fuese ultimada, ella misma sería contradictoria -cesaría, sería seguida-. Pero no puede serlo". Nominalismo, 225. 
incoativa en la máxima amplitud es la demostración de la existencia de Dios. Así pues, esta demostración no se distingue de la existencia creada. Nótese que no es preciso demostrar que la existencia es creada. La expresión 'es creada' es la referencia de la expresión 'la existencia no es la esencia' a la expresión 'la existencia es la existencia'. Tal referencia es el enlace causal" ${ }^{57}$.

Aquí se puede ver con nitidez que demostrar significa pensar. Pensar la actividad existencial de modo coherente de acuerdo con los primeros principios. No se puede pensar congruentemente un primer principio sin los demás primeros principios y sin que ninguno deje de ser primer principio. Ahora se puede comprender también el modo en que Polo se llama continuador de la diferencia real entre esse y essentia. Polo pretende partir de la misma realidad existencial del esse, no de la actualidad de una esencia. De tal manera que afirma que la demostración de la existencia de Dios "no sería posible si la existencia creada fuese un mero hecho o un acontecimiento extraesencial, puesto que en este caso no podría jugar como referencia demostrativa" ${ }^{58}$.

Esa diferencia entre el ser y la esencia permite caracterizar el ser del universo como comienzo. Por eso, "como actividad, la persistencia es la demostración de la existencia originaria; como carencia de sí mismo, es la admisión de la esencia" ${ }^{\circ 9}$. Para Polo demostrar la existencia de Dios significa pensar la actividad persistente como dependiente de la actividad originaria. Así la relación entre Creador y criatura es a la vez íntima y su diferencia es infinita. Sabemos del origen, aunque no esté a nuestro alcance -como ya se ha dicho-el significado de su actividad existencial en sí misma ${ }^{60}$. Sin embargo, la consideración de la actividad existencial divina es un empeño único en la historia de

${ }^{7}$ El ser, 180.

${ }^{58}$ El ser, 216.

${ }^{59}$ El ser, 201. "Si se abandona el límite mental cabe sostener que la demostración de la existencia de Dios es la existencia extramental. La persistencia demuestra que el Origen es tal, esto es, improyectable hacia fuera, en la medida en que la persistencia, carácter existencial creado, no se pierde; y es justamente no perdiéndolo como se advierte que es creado". El ser, 209.

60 "En el argumento a simultaneo se pueden distinguir dos elementos: el primero, de índole reflexiva, es la misma cuestión de la idea de infinito; el segundo es el intento de llegar a la realidad a partir de esa idea. Ninguno de los dos elementos tiene valor en orden a la demostración de la existencia de Dios. Sin embargo, la existencia de Dios es demostrable. El ingreso en el ámbito extramental, en que tal demostración se cifra, no es un paso del orden ideal al real y, como tal, está asegurado por el método propuesto. Pero con ello nada sabemos acerca de la compatibilidad del pensamiento con Dios. ¿Dios es pensable? ¿En qué sentido el pensamiento humano alude a Dios? ¿Qué significa exactamente el límite mental en orden a la identidad originaria? Estas preguntas no afectan a la demostración de la existencia divina, la cual se mantiene al margen de la cuestión de la idea de infinito o se hace imposible”. El ser, 214. 
la filosofía. La novedad es expresada por Polo de la siguiente manera: "la filosofía tradicional llega a Dios como primer principio. Según mi propuesta, a Dios corresponde por antonomasia el sentido principial del ser, por ser la Identidad originaria" ${ }^{61}$. Y ha sido acompañada de un esfuerzo notable por distinguir el ser de la esencia, que es el carácter analítico del ser que consiste en comenzar, de la actividad existencial que puede describirse como comienzo que no cesa ni es seguido: la esencia no es ninguna actividad existencial, sino el análisis de aquella actividad existencial que no se posee a sí misma, porque no tiene término.

Pero es necesario ser preciso. Y Polo lo es claramente: "Ahora bien, esto no significa, en modo alguno, que en la criatura veamos a Dios. En la advertencia alcanzamos a ver solamente la existencia creada. En ella, Ser Incausado no significa esencia a la que se atribuye existencia. No significa nada divino pensado como tal. Aunque a primera vista ello pueda parecer extraño, es perfectamente natural. Nótese que en la advertencia del ser, Dios no puede ser conocido directamente, no solamente porque la advertencia lo es de existencia causada, no de existencia originaria (un respecto incoativo no es el Origen, sino su demostración), sino también porque un conocimiento de Dios concurrente con el conocimiento de la criatura, o que lo prolongue, entraña la suposición de ambos y, por lo tanto, la pérdida de la advertencia... Dios $y$ la criatura no son una totalidad. La advertencia de la existencia creada no se incluye en la existencia de Dios como la parte en el todo. Por lo mismo, la noción de Ser Incausado no es susceptible de consideración independiente en orden a la demostración de su existencia. Al margen de la causa no puede hablarse de actividad; y esto en el preciso sentido de que la actividad no se constituye terminada, no se acaba antes de la referencia... En la correcta comprensión del principio de causalidad se muestra la imposibilidad de un acceso independiente a la existencia de Dios" ${ }^{\prime 2}$.

${ }_{61}$ Epistemología, 192.

62 "Conviene, insisto, poner de relieve que si el efecto es exterior a la causa, la causa no es trascendental. Lo cual ocurre precisamente cuando formulamos objetivamente el principio de causalidad. La cuestión es pertinente para el realismo tomista. En la segunda vía que Tomás de Aquino propone para demostrar la existencia de Dios, aparece la noción de causa primera, o causa incausada. La primera causa es única, pero también es causa de efectos. ¿Este planteamiento es estrictamente correcto? Para responder a la pregunta hemos de apelar al conocimiento habitual: ¿la noción de efecto es compatible con la primariedad del ser? Parece que no, pues el efecto no es primero. Esta dificultad es muy seria. ¿No ocurrirá más bien que el principio de causalidad trascendental nada tiene que ver con la noción de efecto? Como es claro, no tener nada que ver 
De este modo alcanzamos el objetivo: "la advertencia (del ser persistente) culmina en la noción de Incausado. La existencia de Dios es demostrada, pero no como término supuesto de la advertencia, sino en cuanto investida de todo el valor metódico de la primera dimensión del abandono del límite mental" ${ }^{3}$. Es alcanzado el objetivo y su mismo valor intelectivo: "La intimidad del Ser originario es inaccesible porque, si demostrar el carácter existencial del Origen es remover el dato mental, no puede decirse que con ello el Origen sea, propiamente, traído a comparecer" ${ }^{64}$. Por eso, "la existencia originaria se demuestra con la existencia creada, y no en la forma de una profundización o de una atribución a partir de alguna determinación divina pensable" ${ }^{65}$.

Ahora bien, Polo sabe exactamente lo que ha demostrado. "Respecto de Dios, la demostración no dice nada. Entiéndase bien: cuando se sostiene que Dios es el Ser Incausado, pero que esta noción no es una determinación esencial y que se reduce existencialmente al Origen, se confiesa que no es posible decir lo que la noción de Origen significa en Dios, como Dios. Hay que negar que en la demostración se desvele el sentido íntimo de la existencia divina, que sólo es vislumbrada. Que signifique existencia en Dios, como Dios mismo, pertenece a la esfera del misterio" ${ }^{66}$.

Eso tiene un significado preciso para deslindar la teología natural del conocimiento de Dios que se alcanza mediante la fe: "el contenido de la Fe no es sino 'lo que no se sabe al demostrar'. Y esto significa que la demostración no es relativa a la Fe; o lo que es igual, que el Misterio es una noticia y no la ausencia de dato" ${ }^{\prime 67}$.

La demostración, ciertamente logra su objetivo y alcanza la meta: Dios existencialmente significa origen, y así, aunque no podemos alcanzar su esen-

con la noción de efecto no quiere decir admitir la causa sui, sino advertir habitualmente que el principio de identidad no es el de causalidad. Hay tres primeros principios. La solución al problema del efecto tiene que venir por el conocimiento habitual del principio de causalidad trascendental, y por ahí tiene que venir la exclusión de la solución espinozista, puesto que causa sui es una manera objetiva de entender la identidad. Qué curiosa noción de sustancia, por otra parte. Recuérdese que Aristóteles también dice que la sustancia es causa”. Nominalismo, 200-201.

${ }^{63}$ El ser, 215.

${ }^{64}$ El ser, 215. "El Origen es el sentido existencial de la identidad. El sentido existencial de la identidad es inaccesible (sólo cabe demostrarlo incoativamente) porque no sabemos cómo puede culminar originariamente la identidad”. El ser, 249.

${ }^{65}$ El ser, 216.

${ }^{66}$ El ser, 216.

${ }^{67}$ El ser, 229. 
cia como Dios, al menos podemos vislumbrar la intimidad divina. La tarea de la metafísica no es eliminar los misterios sino reconocerlos cuando aparecen y explicar en qué consiste el misterio y cómo podemos alcanzar a vislumbrarlo. Gracias a Dios una demostración de la existencia de Dios no es ni puede ser una varita mágica o aquello que arroje luz suficiente para todo: "la existencia de Dios es demostrable, pero no se puede profundizar en ella" ${ }^{\text {. }}$.

Como en toda demostración, en el trabajo de Polo se manifiesta el esfuerzo de dar cuenta y razón, es decir, la demostración de la existencia de Dios es una tarea intelectual e intersubjetiva, no se hace para uno mismo ni con otra intención que alcanzar la verdad. Y el ámbito que reúne el esfuerzo mental y su comunicación lingüística es la metafísica, la ciencia del ser y de los primeros principios. Esta demostración sólo puede manifestar su importancia al hombre que busque lo primero y en la medida en que comprenda el desarrollo de la filosofía perenne y el fracaso de la filosofía de la subjetividad. Por esa misma razón, las objeciones que diferentes autores han puesto a las demostraciones de la existencia de Dios no se pueden presentar de modo coherente en este contexto, en la misma medida en que resulta una propuesta todavía novedosa, que nos desafía a seguir pensando en profundidad.

\section{b) La demostración antropológica de la existencia de Dios}

Ahora corresponde analizar la demostración de la existencia de Dios desde la antropología trascendental. "La antropología trascendental es el estudio del ser personal, el cual ha de ser investigado con insistencia para que la propuesta no se limite a ser un anuncio; en ella ha de afrontarse también el tema de Dios... Dios es un ser personal con el que el hombre coexiste. Dios es el creador de la persona humana... En atención al ser creado, crear no significa siempre lo mismo. Por eso, el hombre como camino a Dios se distingue de las vías demostrativas de la existencia de Dios propias de la metafísica" ${ }^{\circ}$.

Si crear no significa siempre lo mismo en relación a lo creado, entonces el sentido del ser principial originario deberá ser desarrollado para investigar si es posible un conocimiento más profundo de la existencia de Dios a partir de la antropología trascendental. "Si en metafísica Dios se advierte como Identidad originaria, en antropología esa advertencia ha de ser conservada y reca-

\footnotetext{
${ }^{68}$ El ser, 216.

${ }^{69}$ Epistemología, 192.
} 
bada, pues Dios como transcendens es, asimismo, Originario. Ahora bien, como transcendens a la coexistencia, Dios ha de ser Originariamente Persona. Si lo que caracteriza a la persona humana es la ausencia de réplica, en la Persona Originaria esa carencia no puede tener lugar. Desde luego, la noción de réplica del Origen constituye un misterio: no puede tratarse de 'otro origen', pues ello es incompatible con la identidad. Debe tratarse de una persona distinta"

Obsérvese el paralelismo y la diferencia: en metafísica la advertencia del ser como comienzo sólo puede pensarse con el ser como origen y la causalidad como ligamen trascendental, en la antropología el carácter existencial del co-ser personal no puede pensarse en soledad, el ser personal que no es íntimamente acompañado por otra persona coexistencialmente finita, no puede pensarse sin alcanzar el ser personal del origen trascendental. La demostración, aquí como antes en la metafísica, se reduce al mínimo proceso argumentativo que permita progresar al pensamiento congruentemente.

En un sentido semejante, Polo describe el descubrimiento aristotélico del acto de un modo que puede ayudarnos a comprender las demostraciones de la existencia de Dios. "Aristóteles no dice exactamente que conocer sea un acto, sino que descubrir lo que significa acto es lo mismo que descubrir lo que significa conocer. Energeia no es un predicado del conocer, sino que energeia equivale a conocer. No es que descubramos la índole del conocimiento a la luz de la noción de acto, sino al revés: nos damos cuenta de qué es acto cuando nos damos cuenta de qué es conocer. Y esto porque conocer significa necesariamente estar conociendo: lo conocido es conocido en simultaneidad con el conocer, y no un término por alcanzar o al que llegar... [E]n la consideración de lo que significa conocer se descubre que lo conocido se da en tanto que conocer está siendo... Para Aristóteles el acto no es una etiqueta del conocer. Se justifica cuando uno se percata de lo que es conocer" ${ }^{\prime 1}$.

Como puede verse, se trata de un descubrimiento intelectual que aúna todos los elementos que están en juego, los articula y los presenta de modo coherente entre sí. Pero esos descubrimientos surgen precisamente de la pro-

${ }^{70}$ Epistemología, 195. Ya en El acceso al ser afirmaba que "el saber acerca de la trascendencia está regulado por los primeros principios. Los primeros principios son discernibles de la objetividad. Los primeros principios son el saber regulativo del ser... Los primeros principios susceptibles de desarrollo son dos: el de no contradicción y el de causalidad. El principio de identidad ofrece una problemática especial... no es susceptible de desarrollo sin una ampliación de la noción de trascendentalidad... Esta ampliación requiere una investigación antropológica”, 273.

${ }^{71}$ Introducción, 79-80. 
fundización que tiene lugar cuando se ponen en marcha cada vez más recursos propios, más capacidades ${ }^{72}$.

En el caso de la antropología, Polo acierta a poner en primer lugar la cuestión del quién soy. Cada persona es su ser. Es, en realidad, un co-ser, un ser que coexiste con el universo y con los demás. El ser que puede describirse como además. Precisamente es el ser que se abre por dentro para poder existir con todo. Pero ese ser no tiene en su intimidad ninguna persona en la que mirarse y a la que amar. Como el quién es cada cual y no puede entenderse en la soledad, de ese modo se vive a la búsqueda de aquel que pueda aceptar el ofrecimiento de la propia amistad de manera definitiva. Pero eso sólo puede hacerlo quien es el mismo origen de la persona, porque cada persona muestra que es esencialmente hijo. Si la advertencia del ser principial se orientaba al ser como origen trascendente; así el progreso hacia el propio ser personal conduce a la consideración de la persona trascendente. " $\mathrm{Al}$ asomarse al misterio, la antropología descubre que la carencia de réplica de la persona creada significa que su intimidad no es ninguna 'otra' persona... Dios no es aquello que el hombre no se atreve a pensar como sí mismo, sino que se distingue de él como trascendencia. No existe ningún término de la actividad humana con carácter de persona; la coexistencia carece de término"”3.

Por eso Polo rechaza las fórmulas habituales que emplean con facilidad la palabra demostración: "Se suele decir que la inteligencia es infinita, ya que una inteligencia finita es una contradicción. Por tanto, la inteligencia tiene que ser capax Dei-lo que a veces se propone como una prueba de la existencia de Dios-. Ahora bien, que la inteligencia sea capax Dei es distinto de averiguar el acto por el que se conoce a Dios; hablar de ser capaz de Dios como pura potencia o sin averiguar el acto correspondiente, no pasa de ser una receta. Pero la filosofía no es un mero registro de fórmulas: como vademecum es sumamente tediosa" ${ }^{\text {"74. }}$.

La demostración antropológica de la existencia de Dios es un verdadero descubrimiento filosófico, quizá porque Polo se ha tomado en serio hasta el final el ser de la persona. Por eso, no deja de advertir la diferencia entre ambas demostraciones que estamos estudiando. "El hábito de sabiduría connota que el tema conocido por el intelecto personal es superior al propio intelecto. Con

\footnotetext{
Cfr. Introducción, 44.

73 Epistemología, 195.

74 Antropología I, 155.
} 
otras palabras, el conocimiento de Dios por la persona humana transciende la tercera dimensión del abandono del límite mental. El conocimiento por parte de Dios de la persona humana es imprescindible para que la persona humana conozca a Dios y, por tanto, supera al que la persona alcanza de sí por sí. La demostración de la existencia de Dios en antropología se expresa de esta manera: si Dios no conociera al hombre, no cabría hablar de intelecto humano como co-actus. Esto es una implicación de la distinción real: el hombre no puede conocerse por sí, porque en él el conocer como ser equivale a la persona, y la persona humana como co-actus o co-existencia no es realmente idéntica"75.

En este texto se aúna el carácter diferentemente trascendente de Dios en la metafísica y en la antropología con los actos y hábitos intelectuales necesarios para mostrar este conocimiento como verdadero. El comenzar exigía un origen, ahora la no-identidad de cada quien exige un Dios personal, que me conozca, para que yo que no soy mi conocer, pueda realmente conocer y trascenderme.

\section{CONCLUSIÓN}

A mi entender la distinción entre metafísica y antropología que sirve para diferenciar estas dos demostraciones de la existencia de Dios tiene que ver con el esfuerzo por pensar tanto el problema de la conexión aristotélica del primer motor inmóvil como su caracterización como intelecto que se entiende a sí mismo. Por eso, Polo en Introducción a la filosofía expone un argumento aristotélico para destacar la importancia de la dimensión antropológica de la teología natural. "Aristóteles llega a Dios... (por) la consideración del carácter intermitente que tiene en el hombre la teoría, es decir, el ejercicio de los actos intelectuales. El hombre no siempre está en acto de conocer. Si existe un ser que teoriza sin intermitencia, que está siempre en acto de entender, que es enteramente noús en acto, hay que llamarle Dios. Es un trascender desde la potencia y el acto en el orden del noús (recuérdese la dualidad de los sentidos del acto. Considerado precisivamente, el primer motor podría ser entendido como sustancia. En cambio, el trascender a lo primero en el orden del noús no

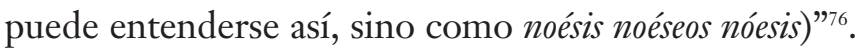

75 Antropología I, 148.

${ }^{76}$ Introducción, 116. Y más adelante, en la misma página, añade. "Propiamente, se llega a Dios considerando el modo de vida que es la intelección. Si desde la vía del movimiento físico no se 
Por eso justamente Polo necesita profundizar en la distinción real tomista entre esse y essentia: es decir la inidentidad del ser finito y del hombre que piensa. La filosofía clásica solía unir ambos argumentos con la demostración del carácter intelectual de cualquier espíritu. Pero ésa era una manera indirecta de unir la filosofía de la naturaleza y la antropología. Polo consigue demostrar el carácter metafísico de la inquisición por el ser, de tal forma que la demostración no dependa de premisas cosmológicas discutibles. Por otro lado, consigue demostrar el carácter transcendental de la investigación antropológica sobre la persona. De ese modo, la demostración conduce directamente al carácter personal de Dios, que anteriormente debía ser demostrada indirectamente aduciendo premisas adicionales cuyo estatuto epistemológico podía ser dudoso. Y, correspondientemente, también afianza el carácter personal de todo ser humano: "La libertad no es una prueba de la existencia de Dios, sino de la personalidad de Dios. Pero también es una prueba de la personalidad de los demás hombres" ${ }^{\prime 7}$.

En este itinerario de la vida intelectual humana en busca de Dios lo que se ha obtenido es principalmente crecer, que es lo más propio del ser humano. Crecemos porque apuntamos a lo más alto, porque no nos conformamos con un saber limitado. "Ver a Dios es el tener que se busca en cuanto que la libertad está otorgada en el orden de la plenitud"78. Buscando a Dios el hombre encuentra quién es él porque entrevé a aquel que le trasciende, de quien depende porque le ha creado y espera que aceptemos el regalo y le ofrezcamos el regalo de vivir de acuerdo con lo divino que hay en nosotros. El amor personal donal es "la asimilación creatural al Hijo de Dios. Al ser inabarcable la aceptación divina, ésta constituye en antropología la guía hacia lo insondable" "79. Esa guía es tal, por eso "la búsqueda depende de la futuridad libre" Sólo desde la cumbre se atisba la belleza de todo el camino recorrido y reconocemos que ha valido la pena el ejercicio de la libertad que ha supuesto.

concluye sobre el ser de Dios más que de una manera oscura, a partir de la teoría (la más alta forma de vida, cuyo ejercicio es superior a la acción física), conviene decir que si el hombre es intelectual en potencia y acto, el acto de entender al margen de cualquier potencia es la vida perfecta, la protovida, la razón primera de la vida (esto lo desarrolla Aristóteles, sobre todo, en los libros IX y XII de la Metafísica). Decir que Dios existe es lo mismo que decir que Dios vive.

Y es la plenitud de la vida como plenitud de la teoría (entenderse siempre en acto)".

77 Quién es el hombre, 223.

78 El acceso, 112.

79 Epistemología, 196.

${ }^{80}$ El acceso, 111. 